Journal of Al- Azhar University Engineering Sector

Vol. 14, No. 53, October, 2019, 1594-1605

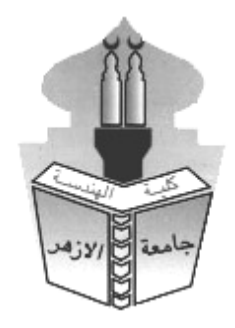

\title{
PREPARE BIM PROJECT EXECUTION PLAN
}

\author{
Abdelhakim Mohamed Talaat \\ Department of Architecture- Faculty of Engineering, Ain Shams University, Cairo, Egypt \\ E-mail: abdelhakim@tasmem-eg.com
}

\begin{abstract}
After project agreement and before Starting BIM process, it is recommended to prepare a BIM project execution plan ( $\mathrm{PxP}$ or $\mathrm{BxP})$.The $\mathrm{BIM}$ plan should outline the overall vision with implementation details to be as a clear guide to all project stakeholders. The BIM Execution Plan is a living document should be developed by the project BIM manager and refined throughout the project's life cycle.There are some steps procedure to develop a detailed BIM plan, starting from identifying the appropriate BIM goals and uses, designing BIM process maps, and identifying the supporting infrastructure to successfully implement the plan. The plan should be prepared by designer lead company in accordance with all parties'needs, and should be a restricted reference to be followed by all.
\end{abstract}

KEYWORDS : BIM, Project execution plan, PxP, BxP, Coordination, Information Exchange, Consultants firms, Designers.

$$
\begin{aligned}
& \text { إعداد خطة تنفيذ المشروع بواسطة نمذجة معلومات البناء } \\
& \text { عبدالحكيم محمد طلعت } \\
& \text { قسم الهندسة المعمارية، كلية الهندسة، جامعة عين شمس، القاهرة ، جمهورية مصر العربية }
\end{aligned}
$$

قبل البدأ في تنفيذ أي مشروع بو اسطة نمذجة معلومات البناء وبعد توقيع العقد مباشرة يجب وضع خطة الإنة عمل واضحة وشاملة

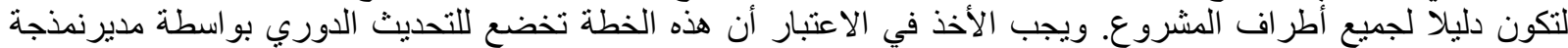

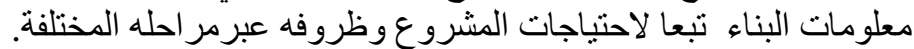

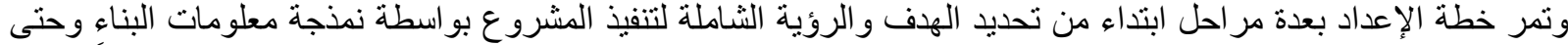

صياغة خطة تنفيذ المشروع بنمذجة معلومات البناء لتكون بمثابة مرجع موحد لجميع أطرف المشروع يلتزمون بهن به جميعاً.

الكلمات المفتاحيه : نمذجة معلومات البناء BIM ، خطة عمل BxP ، PXP ، التسيق ، الريفيت ، تبادل المعلومات ،

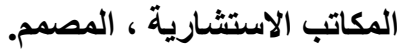

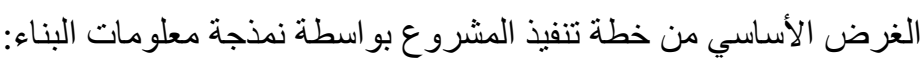

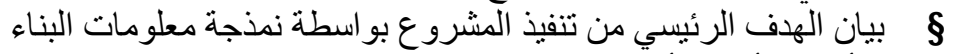

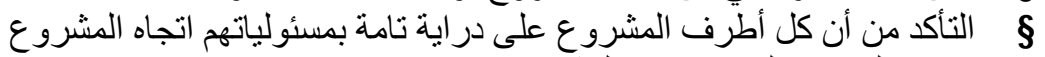

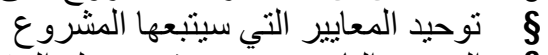

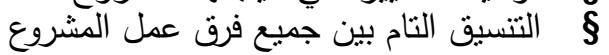




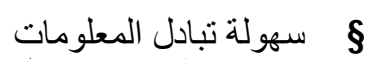

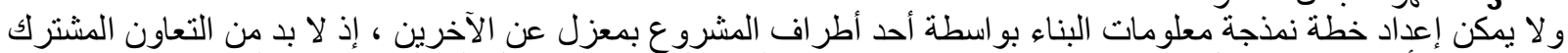

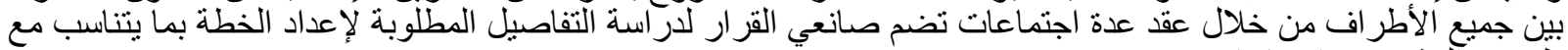
ظروف ألمشروع و احتياجاته.

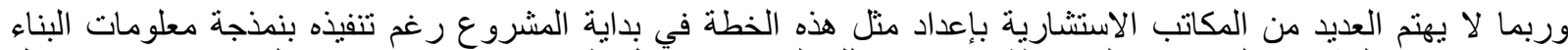

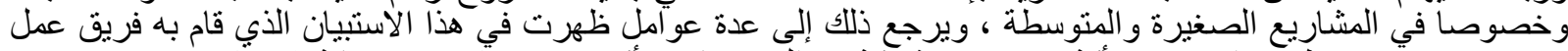

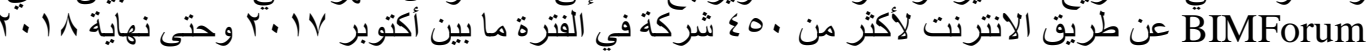

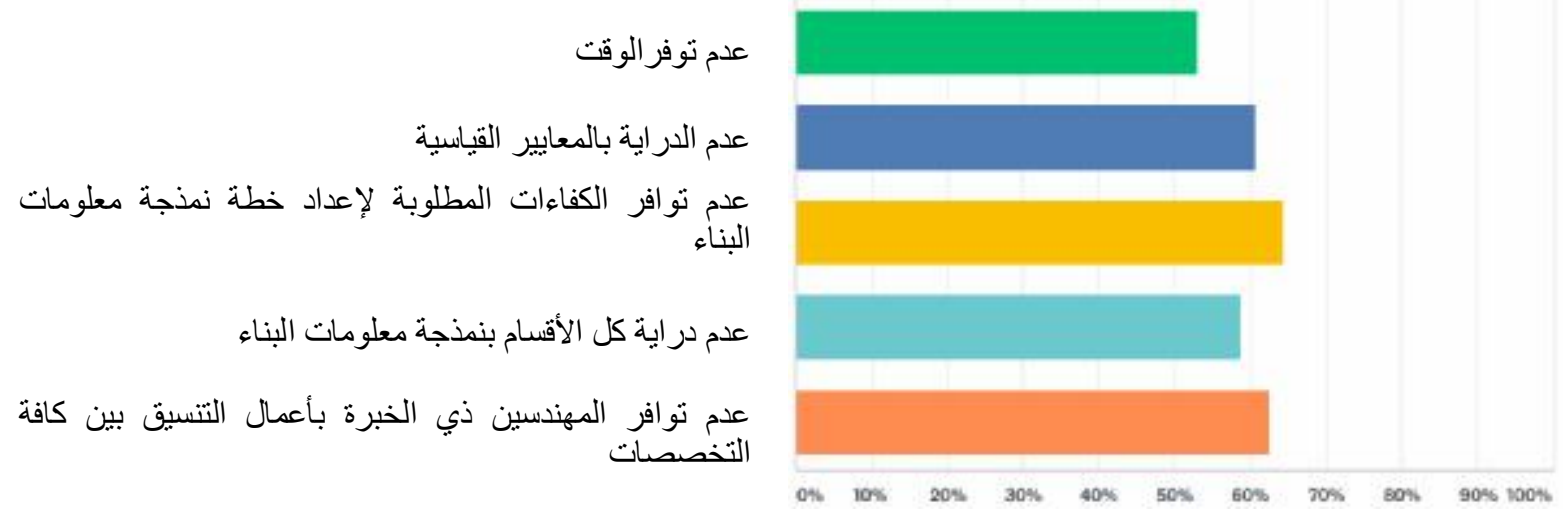

شكل 1 : نتيجة السؤال عن سبب عدم استخدام خطة نمذجة معلومات البناء في المشروعات الصغيرة والمتوسطة في الولايات المتحدة

BIMForum BxP Guide V0.00 Page 65

التفكير في النهاية منذ البداية

لتطبيق نمذجة معلومات البناء بنجاح لا بد من النظرة بعيدة المدى ، حيث إنه من الضروري بمكان البهان أن يستو عب فريق العمل

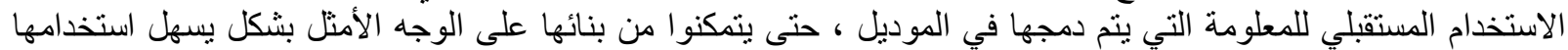

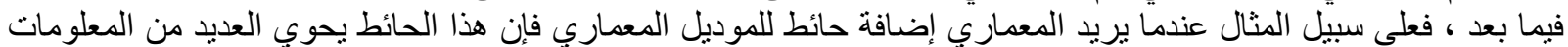

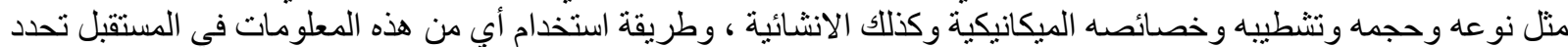

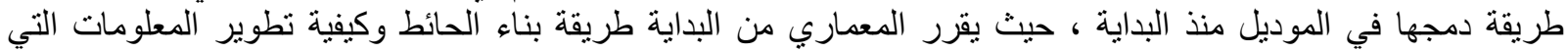
تصاحبه عبر مر أحل المشروع. المراجع الدولية لخطة عمل نمذجة معلومات البناء

يوضح الجدول التالي أثهر المراجع الدولية في هذا الإطار ومقارنة سريعة بينهما.

\begin{tabular}{|c|c|c|}
\hline BIM Project Execution $\begin{array}{r}\text { Plan } \\
\text { Guide }\end{array}$ & $\begin{array}{rr}\text { Project Execution } & \begin{array}{r}\text { Planning } \\
\text { Guide }\end{array}\end{array}$ & اسم المرجع \\
\hline Version 0, September 2018 & Version 2.1, May 2011 & آخر آصدارها نسخة تم \\
\hline BIM Forum & Building Smart Alliance / NBIMS-US & جهة الإصدار \\
\hline $\begin{array}{r}\text { BxP } \\
\text { BIM Execution Plan }\end{array}$ & $\begin{array}{r}\text { PxP } \\
\text { BIM Project Execution Plan }\end{array}$ & الاختصار المعتمد \\
\hline ورما يجز على ذكر محتويات خطة العمل نفسها & 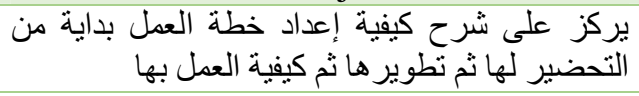 & المحتويات \\
\hline
\end{tabular}



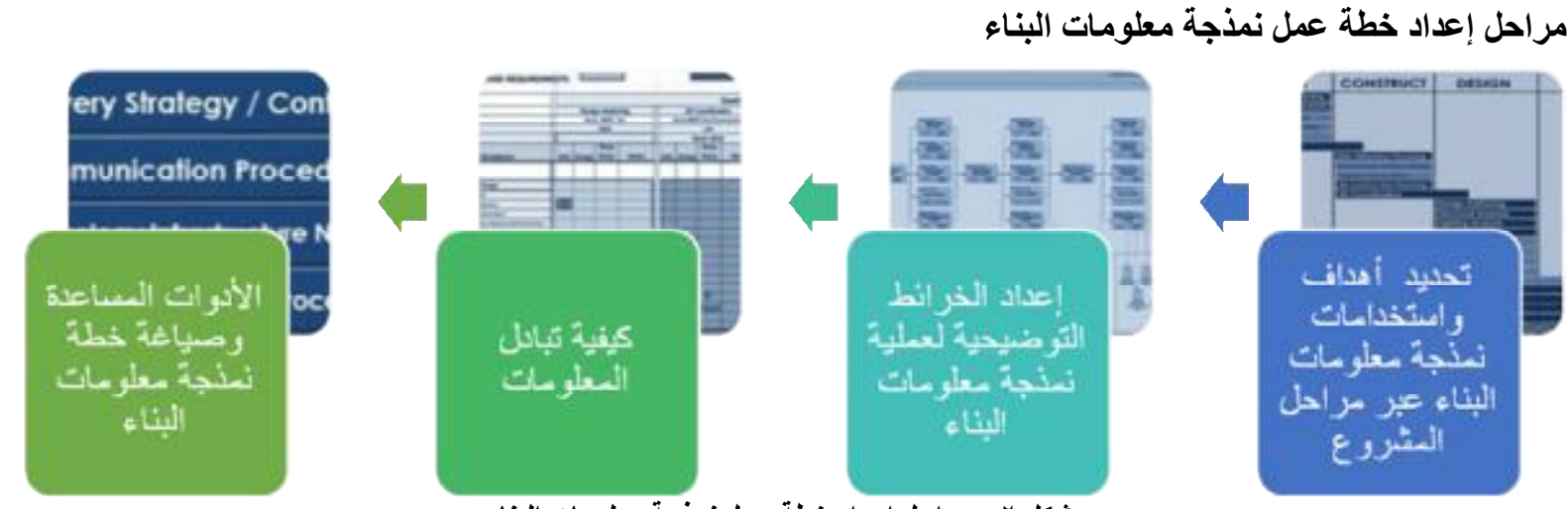

شكل ؟ : مراحل إعداد خطة عمل نمذجة معلومات البناء

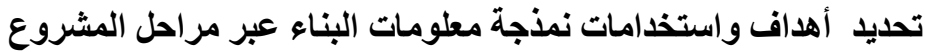

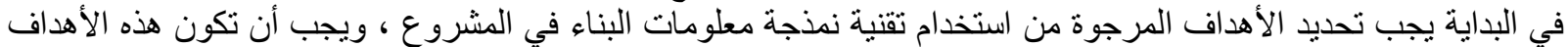
خاصة بالمشروع وليست عامة ، فتكون واضحة ومحددة وقابلة للقياس ومن الأمثلة على الأهداف المرجوة من استخدام نمذجة الأنة

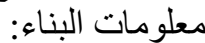
تقليل المدة الكلية للجدول الزمني للمشروع B B

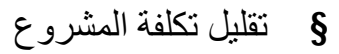

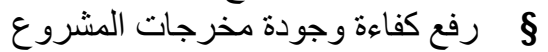

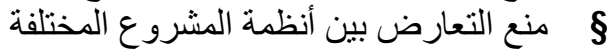

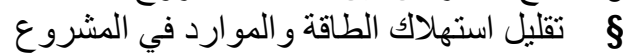

وبمجرد الانتهاء من تحديد أهداف استخدام نمذجة معلومات البناء في المشروع يبدأ بعدها فريق العمل في تحديد المهام التي

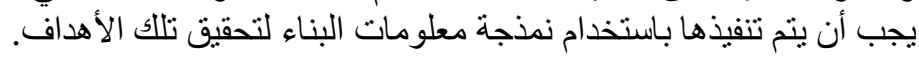

وعن هذه المهام فهنالك ه ب استخداما للبيم عبر مر احل المشروع تم رصدهم من خلال عدة لقاءات و استبيانات مع خبراء صناء صناعة البناء

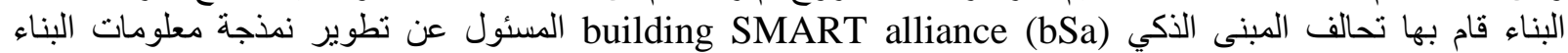
بالو لايات المتحدة الأمريكية ، كما هو موضح في الجدول التالي (الأخضر الغامق للاستخدامات الأكثر أهمية) 
إعداد خطة تنفيذ المشروع بواسطة نمذجة معلومات البناء

جدول r : استخدامات نمذجة معلومات البناء

BIM Project Execution Planning Guide V2.1 Page 12

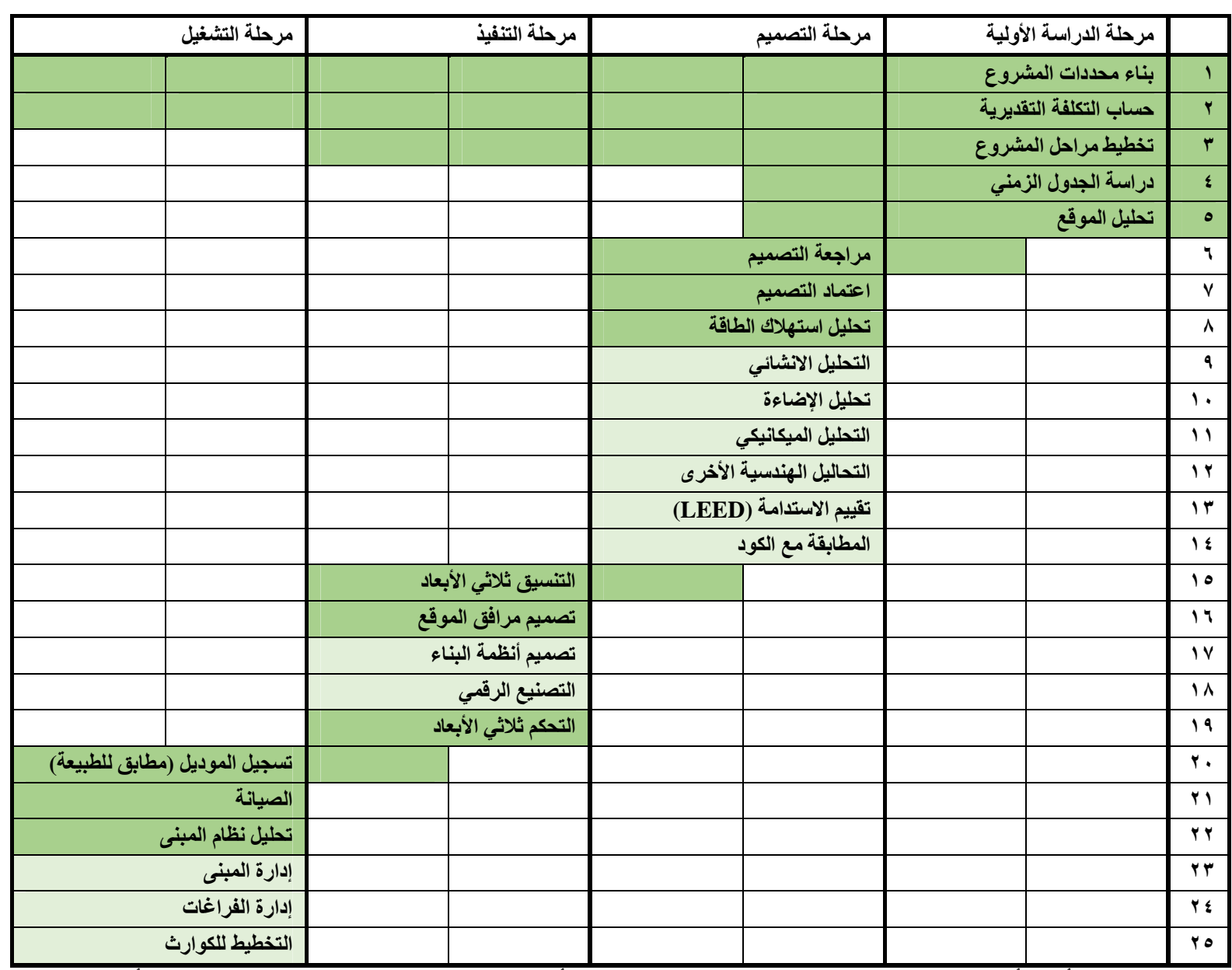

ويجب التنويه أن الأهداف تكون مرتبطة باستخدامات معينة دون أخرى، فعلى سبيل المثال إذا كان من احد أهداف المشروع

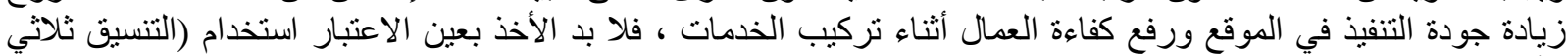

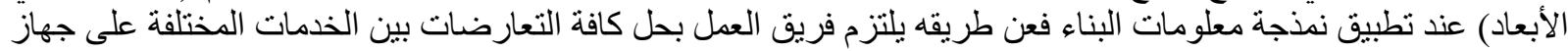

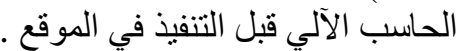

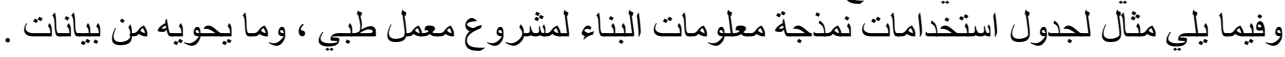


إعداد خطة تنفيذ المشروع بواسطة نمذجة معلومات البناء

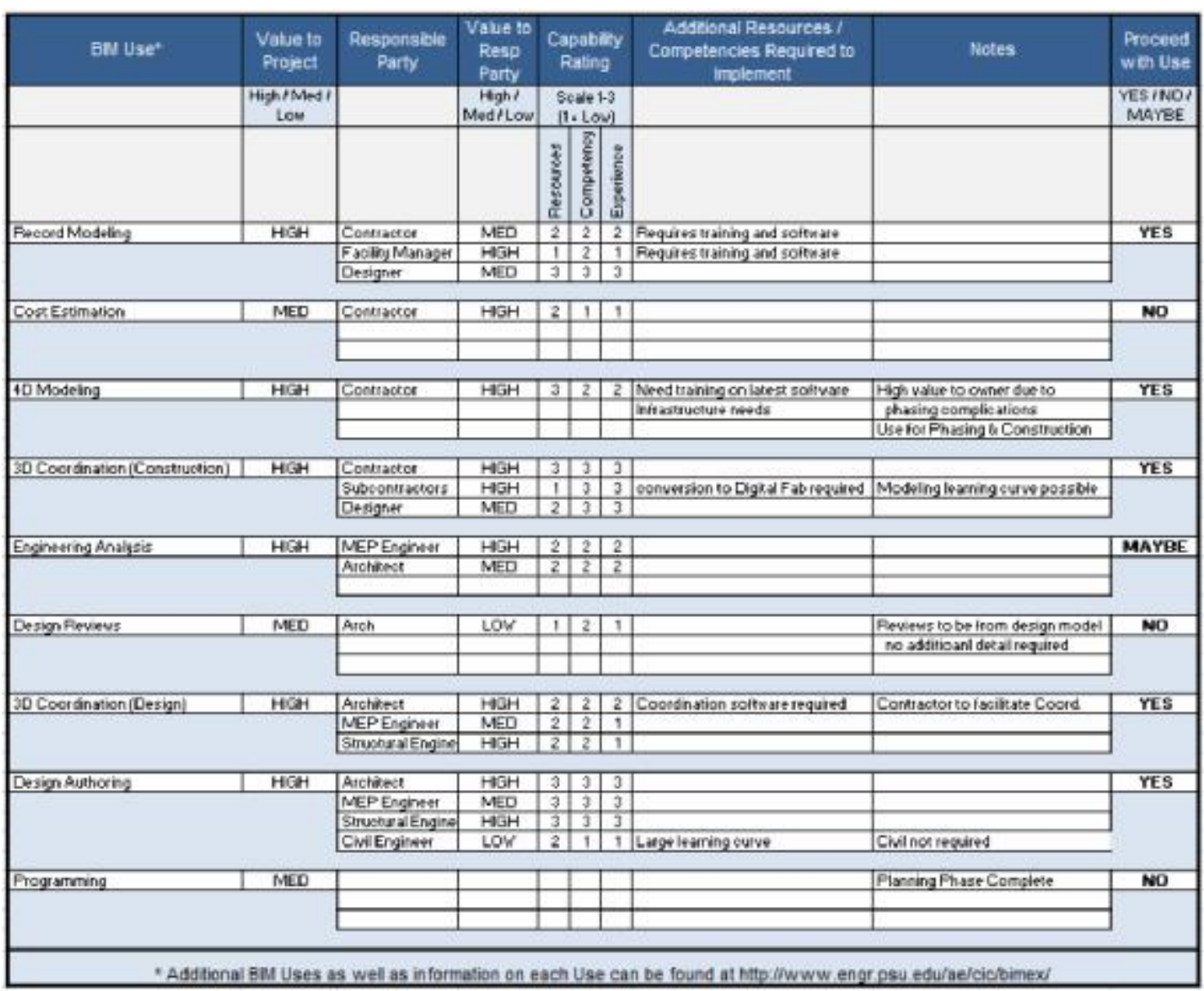

جدول r : استخدامات نمذجة معلومات البناء لمشروع معمل طبي

BIM Project Execution Planning Guide V2.1 Page 13

و ولإعداد مثل هذا الجدول السابق لا بد من اتباع الخطوات التالية:

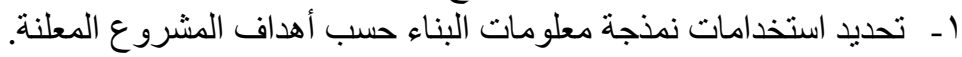

تحديد الجهة المسئولة عن كل استخدام للبيم (المالك / المصمم /

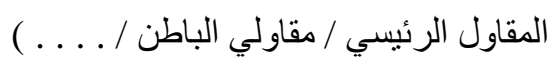

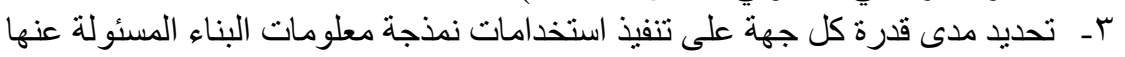

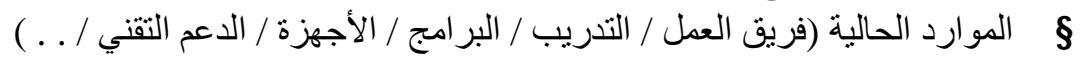

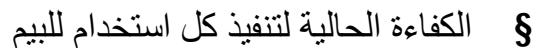

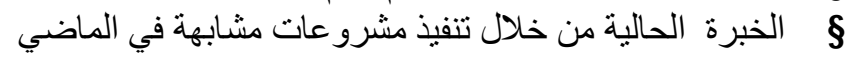

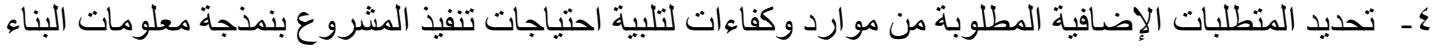

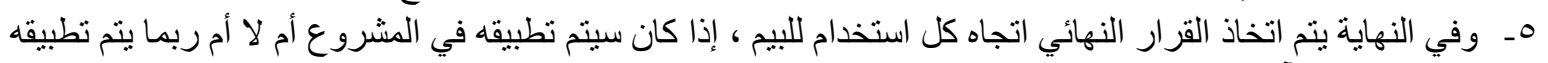

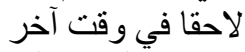

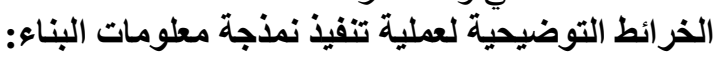

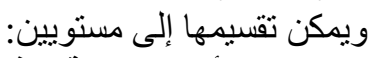

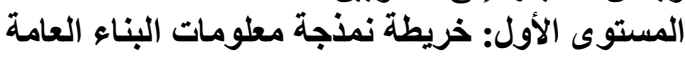

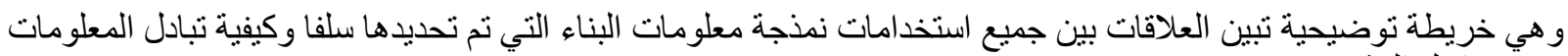

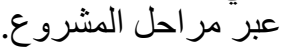




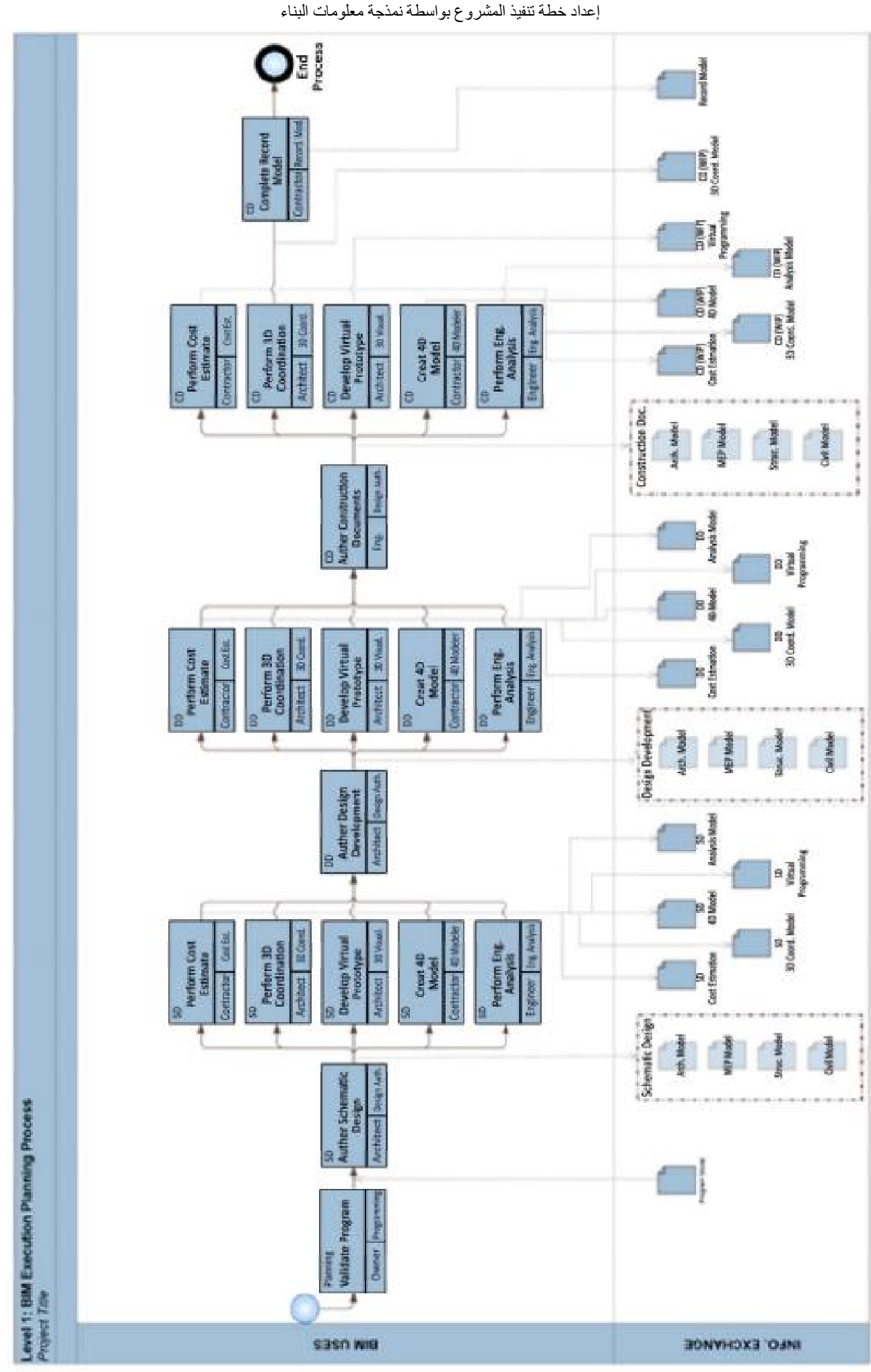

شكل r : نموذج لخريطة نمذجة معلومات البناء العامة

BIM Project Execution Planning Guide V2.1 Page 19 
إعداد خطة تنفيذ المشروع بواسطة نمذجة معلومات البناء

المستوى الثاني: خرائط نمذجة معلومات البناء التفصيلية

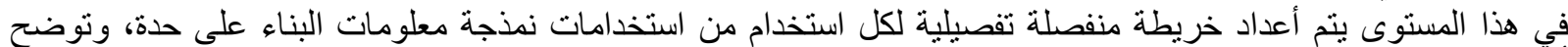
أيضا الجهات المسئولة عن هذا الاستخدام وكيفية تبادل المعلومات بينهم.

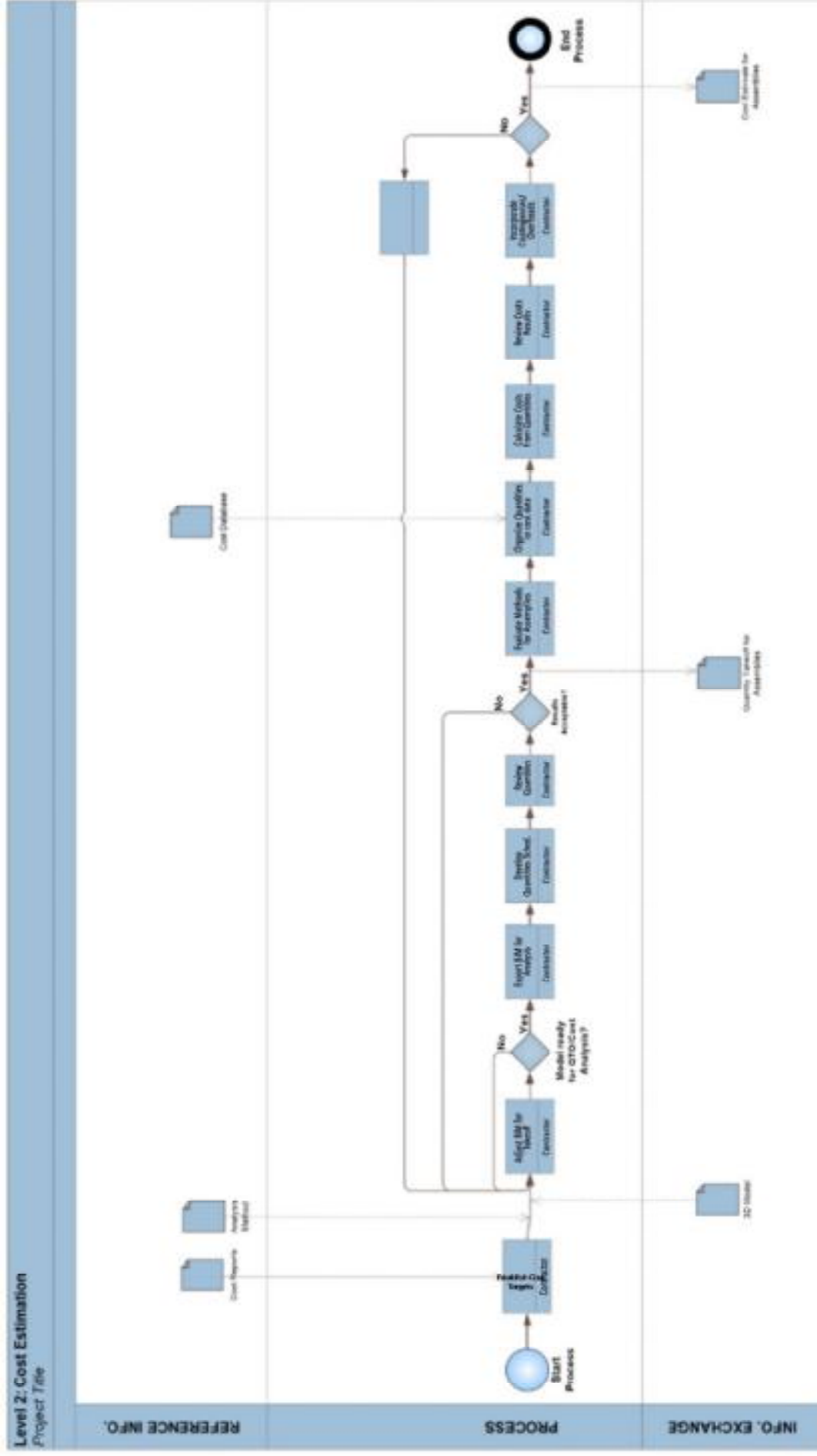

شكل ؛ : نموذج لخريطة نمذجة معلومات البناء التفصيلية للتكلفة التقليرية

BIM Project Execution Planning Guide V2.1 Page 77

1600 
إعداد خطة تنفيذ المشرو ع بو اسطة نمذجة معلومات البناء

\begin{tabular}{|c|c|c|}
\hline Element & Description & Nota \\
\hline Event & $\begin{array}{l}\text { An Event is an occurrence in the course of a business process. Three } \\
\text { types of Events exist, based on when they affect the flow: Start, } \\
\text { Intermediate, and End. }\end{array}$ & \\
\hline Process & $\begin{array}{l}\text { A Process is represented by a rectangle and is a generic term for } \\
\text { work or activity that entity performs. }\end{array}$ & \\
\hline Gateway & $\begin{array}{l}\text { A Gateway is used to control the divergence and convergence of } \\
\text { Sequence Flow. A Gateway can also be seen as equivalent to a } \\
\text { decision in conventional flowcharting. }\end{array}$ & \\
\hline Sequence Flow & $\begin{array}{l}\text { A Sequence Flow is used to show the order (predecessors and } \\
\text { successors) that activities will be performed in a Process. }\end{array}$ & \\
\hline Association & $\begin{array}{l}\text { An Association is used to tie information and processes with Data } \\
\text { Objects. An arrowhead on the Association indicates a direction of } \\
\text { flow, when appropriate. }\end{array}$ & \\
\hline Pool & $\begin{array}{l}\text { A Pool acts as a graphical container for partitioning a set of activities } \\
\text { from other Pools. }\end{array}$ & \\
\hline Lane & $\begin{array}{l}\text { A Lane is a sub-partition within a Pool and will extend the entire } \\
\text { length of the Pool - either vertically or horizontally. Lanes are used } \\
\text { to organize and categorize activities. }\end{array}$ & 1 \\
\hline Data Object & $\begin{array}{l}\text { A Data Object is a mechanism to show how data is required or } \\
\text { produced by activities. They are connected to the activities through } \\
\text { Associations. }\end{array}$ & Name| \\
\hline Group & $\begin{array}{l}\text { A group represents a category of information. This type of grouping } \\
\text { does not affect the Sequence Flow of the activities within the group. } \\
\text { The category name appears on the diagram as the group label. } \\
\text { Groups can be used for documentation or analysis purposes. }\end{array}$ & \\
\hline
\end{tabular}

شكل • : شرح الأشكال المستخدة في خرائط نمذجة معلومات البناء

BIM Project Execution Planning Guide V2.1 Page 22

يجب تحديد المعلومات المطلوبة من كل جهة من أطرات افت المشروع في مرحلة مبكرة ، ويتم ذلك عن طريق ورقة تبادل

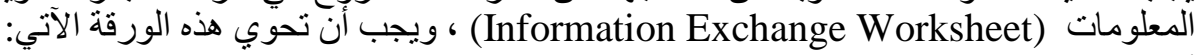
أطر اف المشروع المسئولة عن استقبال المعلومات (Model Receiver) نوع الملفات التي سيتم تداول المعلومات من خلالها (Model File Type) المعلومات الضرورية فقط لاستخدامات نمذجة معلومات البناء التي تم تحديدها (Information) أية ملاحظات أخرى (Notes) 
إعداد خطة تنفيذ المشرو ع بو اسطة نمذجة معلومات البناء

\begin{tabular}{|c|c|c|c|c|c|c|c|c|c|c|c|c|}
\hline \multicolumn{2}{|r|}{ Information } & \multicolumn{2}{|r|}{ Responsible Party } & \multirow{4}{*}{\multicolumn{2}{|c|}{$\begin{array}{c}\text { A } \\
\text { Output }\end{array}$}} & & \multirow{4}{*}{\multicolumn{2}{|c|}{$\begin{array}{c}\text { B } \\
\text { Input }\end{array}$}} & & & & \\
\hline$A_{A}$ & 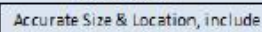 & ARCH & Architect & & & \multirow{4}{*}{$=$} & & & & \multirow{4}{*}{+} & \multirow{3}{*}{\multicolumn{2}{|c|}{$\begin{array}{l}\text { Additional } \\
\text { Information }\end{array}$}} \\
\hline A & materiels and object parameters & $\frac{\mathrm{CON}}{\mathrm{CE}}$ & $\begin{array}{l}\text { Contractor } \\
\text { Civil Engineer }\end{array}$ & & & & & & & & & \\
\hline \multirow[t]{2}{*}{ B } & \multirow{2}{*}{$\begin{array}{l}\text { General Size \& Location, include } \\
\text { parameter data }\end{array}$} & $\mathrm{FM}$ & Facility Manager & & & & & & & & & \\
\hline & & MEP & MEP Engineer & & & & & & & & & \\
\hline \multirow[t]{2}{*}{ c } & \multirow{2}{*}{ Schematic SIze \& Location } & SE & Structural Engineer & \multirow{2}{*}{\multicolumn{3}{|c|}{ A OUTPUT }} & \multirow{2}{*}{\multicolumn{3}{|c|}{$\mathrm{B}^{\prime}$ INPUT }} & \multirow{2}{*}{\multicolumn{3}{|c|}{$C^{\prime}$ INPUT }} \\
\hline & & & Trade Contractors & & & & & & & & & \\
\hline \multicolumn{4}{|c|}{ Information Exchange Title } & \multicolumn{3}{|c|}{ Design Authoring } & \multicolumn{3}{|c|}{ 3D Coordination } & \multicolumn{3}{|c|}{ Energy Analysis } \\
\hline & & & & \multicolumn{3}{|c|}{ OUTPUT } & \multicolumn{3}{|c|}{ INPUT } & \multicolumn{3}{|c|}{ INPUT } \\
\hline \multicolumn{4}{|c|}{ Time of Exchange (SD, DD, CD, Construction) } & & & & \multicolumn{3}{|c|}{$\mathrm{DD}$} & & & $\mathrm{Q}$ \\
\hline Mor & del Reciever & & & & $\mathrm{N}$ & & & & & & & EP \\
\hline Rec & iever File Format & & & & & & & & & & & \\
\hline App & lication \& Version & & & & & & & & & & & \\
\hline & Model Elen & lent Brea & kdown & Info & $\begin{array}{l}\text { Resp } \\
\text { Party }\end{array}$ & Notes & Info & $\begin{array}{l}\text { Resp } \\
\text { Party }\end{array}$ & Notes & Info & $\begin{array}{l}\text { Resp } \\
\text { Party }\end{array}$ & Notes \\
\hline B & SHELL & & & & & & & & & & & \\
\hline & Superstructure & & & & & & & & & & & \\
\hline & & Floor Cons & truction & $\mathrm{B}$ & A & & $\mathrm{B}$ & A & & $\mathrm{B}$ & A & \\
\hline & & Roof Cons & truction & $\mathrm{B}$ & A & & $\mathrm{B}$ & A & & $B$ & A & \\
\hline & Exterior Enclosure & & & & & & & & & & & \\
\hline & & Esterior W/a & & $\mathrm{B}$ & A & & A & A & & $\mathrm{B}$ & A & RV Value \\
\hline & & Esterior Wir & dows & $B$ & A & & $B$ & A & & A & A & Rivalue \\
\hline & & Exterior Do & & $B$ & A & & & & & C & A & \\
\hline & Roofing & & & & & & & & & & & \\
\hline & & Roof Cove & rings & $B$ & A & & & & & & & \\
\hline & & Roof Open & & $\mathrm{B}$ & A & & A & A & & $\mathrm{B}$ & A & \\
\hline C & INTERIORS & & & & & & & & & & & \\
\hline & Interior Construction & & & & & & & & & & & \\
\hline & & Partitions & & $\mathrm{B}$ & $\mathrm{A}$ & & $\mathrm{B}$ & A & & $\mathrm{B}$ & A & \\
\hline & & Interior Doo & & & & & & & & C & A & \\
\hline & & Fittings & & $\mathrm{B}$ & A & & $B$ & A & & & A & \\
\hline & Stairs & & & & & & & & & & & \\
\hline & & \begin{tabular}{|l} 
Stair Const \\
\end{tabular} & ruction & $\mathrm{B}$ & A & & $\mathrm{B}$ & A & & $\mathrm{B}$ & A & \\
\hline & & Stair Finish & & & & & & & & & & \\
\hline & Interior Finishes & & & & & & & & & & & \\
\hline & & Wall Finishe & & & & & & & & $B$ & A & Refleotance \\
\hline & & Floor Finis! & & & & & & & & $B$ & $A$ & Reflectance \\
\hline & & Ceiling Fini: & shes & & & & & & & $B$ & A & Reflectance \\
\hline $\mathbf{D}$ & SERVICES & & & & & & & & & & & \\
\hline
\end{tabular}

جدول ؛ : مثال لورقة تبادل المعلومات (Information Exchange Worksheet) BIM Project Execution Planning Guide V2.1 Page 26

الأدوات المساعدة وصياغة خطة نمذجة معلومات البناء

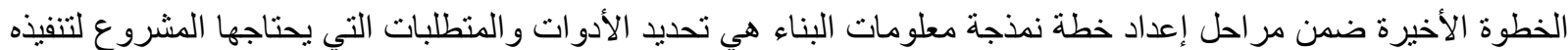

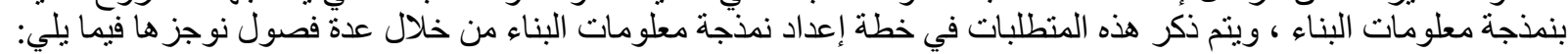
نظرة عامة على خطة تنفيذ نمذجة معلومات البناء

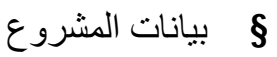
مو اعيد التسليمات الرئيسية في المشروع B

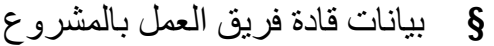
أهداف نمذجة معلومات البناء في المشروع و و استخداماته

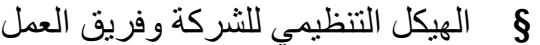

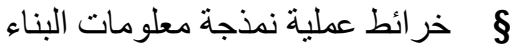

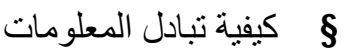
متطلبات المالك من استخدام نمذجة معلومات البناء

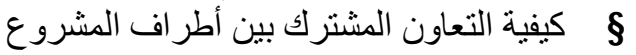

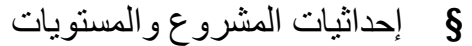
طرق تسمية الملفات و عناصر الموديل و اللوحات م

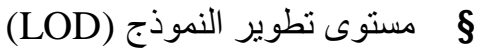




$$
\begin{aligned}
& \text { إعداد خطة تنفيذ المشروع بو اسطة نمذجة معلومات البناء }
\end{aligned}
$$

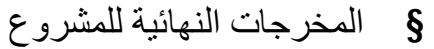

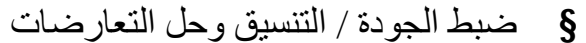

$$
\begin{aligned}
& \text { B المتطلبات التقنية لاستخدام نمذجة معلومات البناء البناء }
\end{aligned}
$$

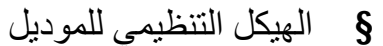

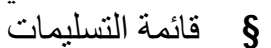

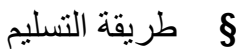

وكما يمر المشروع بعدة مر احل بدءا من الفكرة الأولية حتى التنفيذ والتتشيل فإن مستند خطة التنفيذ هو الآخر يمر بعدة مر احل حيث يتم تطويره باستمر ار ليتمانثى مع طبيعة كل مرحلة.

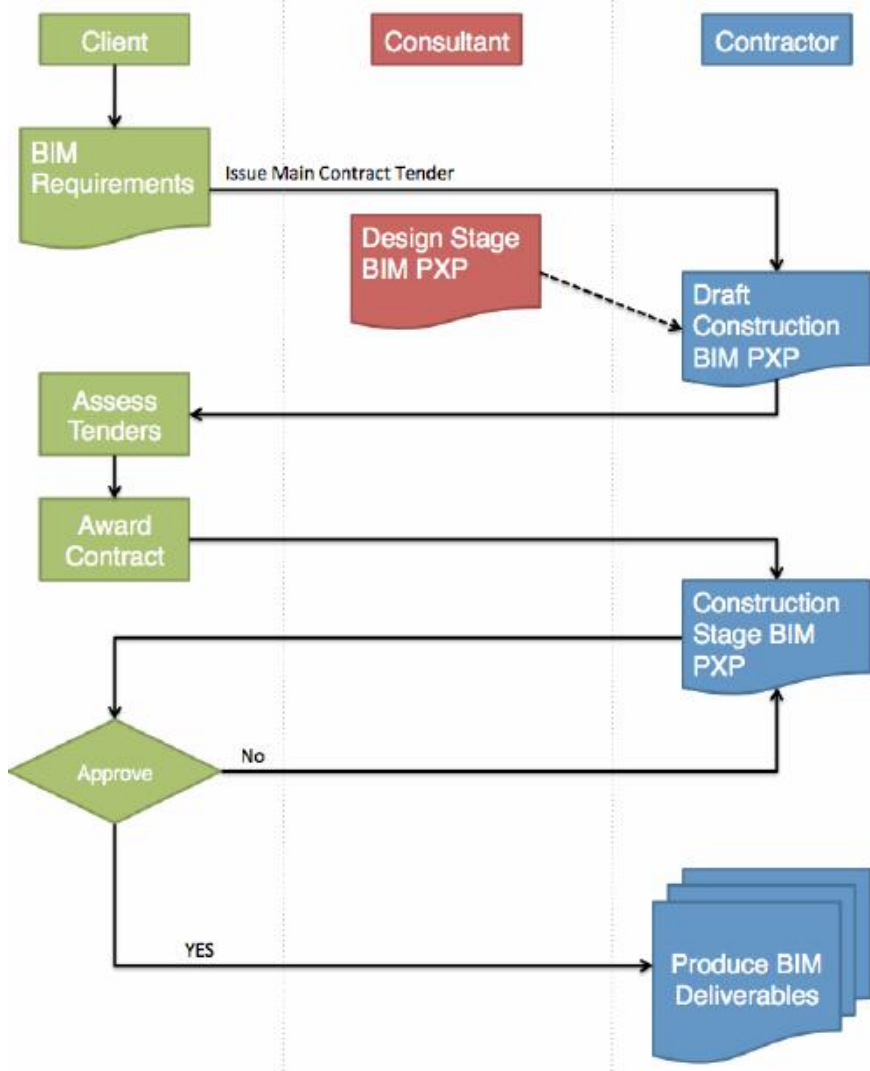

شكل (V) مستند خطة التنفيذ أثناء مرحلة التنفيذ

CIC Building Information Modeling Standards, Page 13

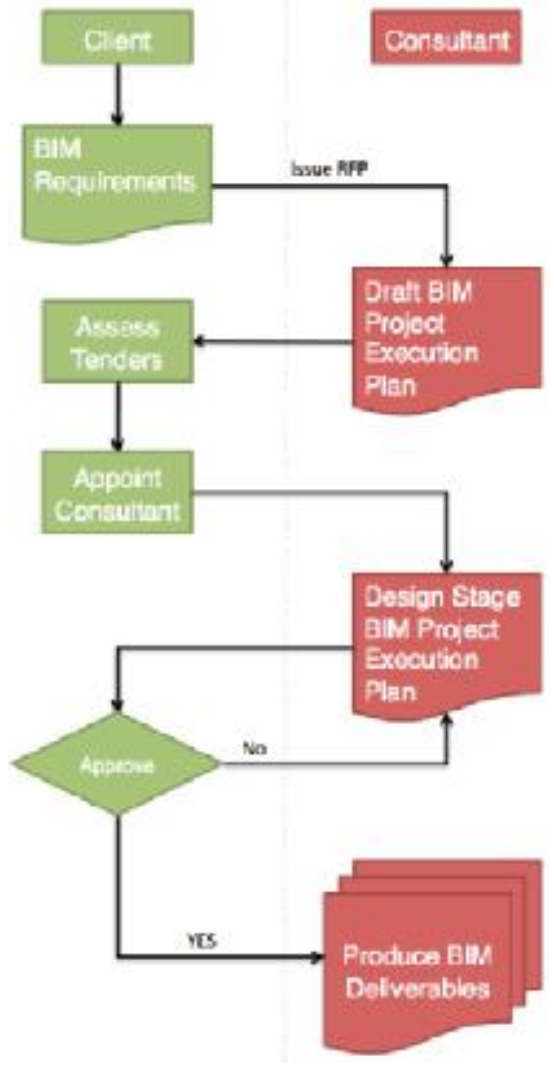

شكل (؟) مستند خطة التنفيذ أثناء مرحلة التصميم CIC Building Information Modeling Standards, Page 12

نماذج خطة تنفيذ المشروع بو اسطة نمذجة معلومات البناء: هناك العديد من النماذج الدولية المعدة مسبقاً لتكون دليلاً إرشادياً لإعداد خطة تنفيذ نمذجة معلومات البناء ومن أنشر هذه

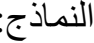

و الذي تم إعداده بواسطة جامعة ولاية بنسلفانيا ، قسم أبحاث كمبيوتر البناء المتكامل Computer Integrated

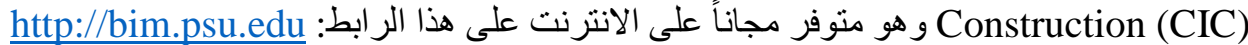


Y. النموذج البريطاني

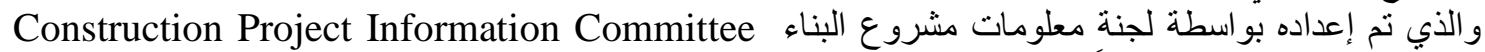
https://www.cpic.org.uk/cpix/cpix-bim- و هو منوفر مجاناً على الانترنت على هذا الر ابط: اعنى (CPIC)

execution-plan

اعتمدت هذه الورقة البحثية على مر اجع دولية اهتمت بوضع معايير قياسية لأي شركة تريد إعداد خطة تنفيذ المشروع بو اسطة

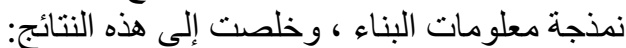

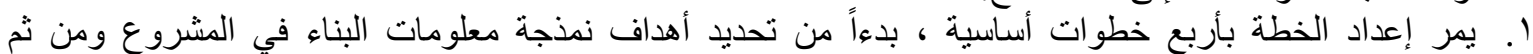

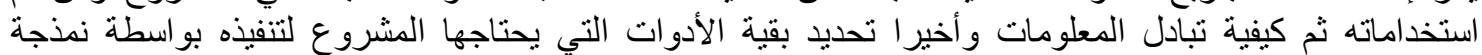

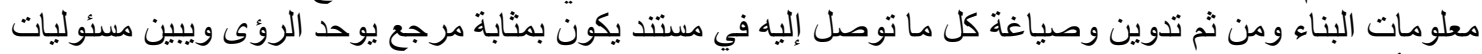

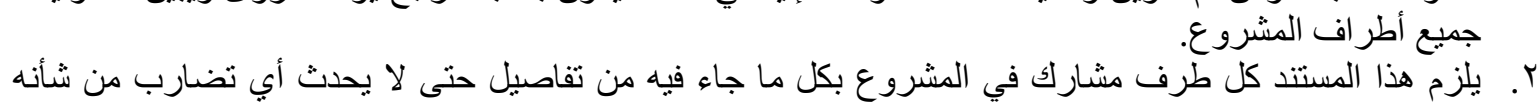

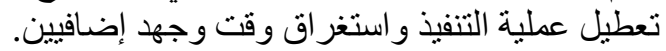

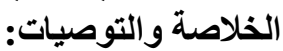

إن أغلب الثركات التي اهتمت بتنفيذ تقنية نمذجة معلومات البناء ثم تعثرت وتر اجعت و وعادت إلى استخدام الأوتوكاد كان سببها

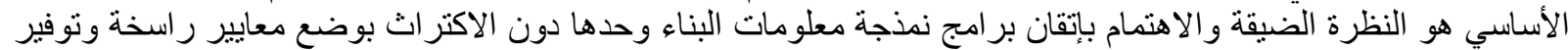

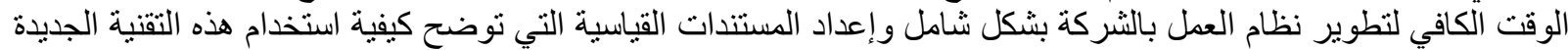

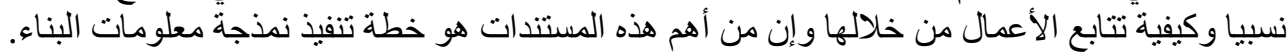

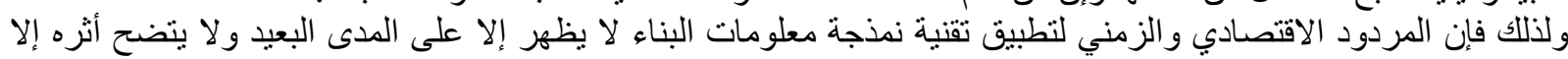

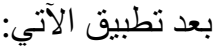

ا. . تطوير نظام العمل بالثركة بالكامل ليتو اعم مع تطبيق المنظومة الجديدة ، وإعداد المستندات القياسية الخاصة بها.

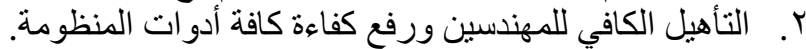

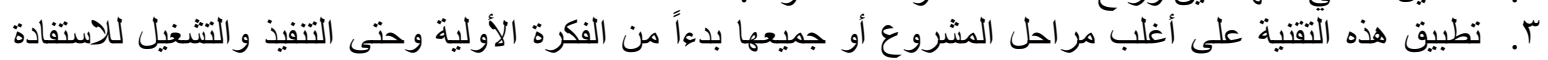

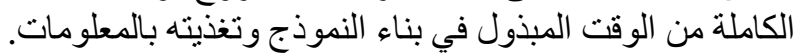

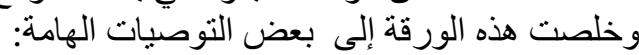

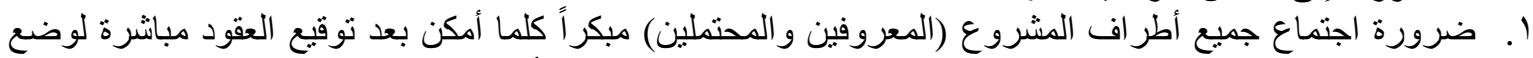

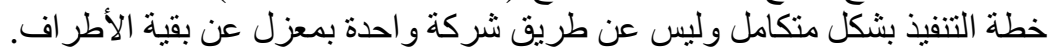

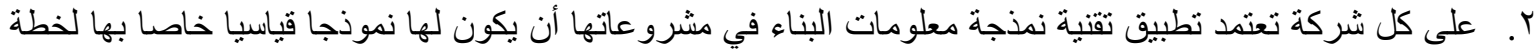

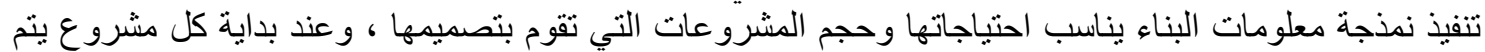

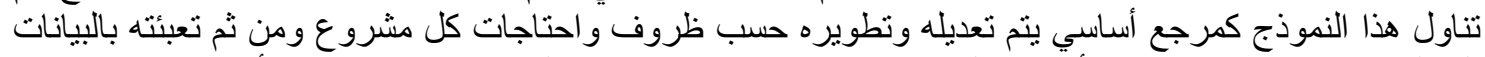

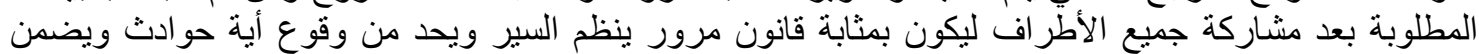

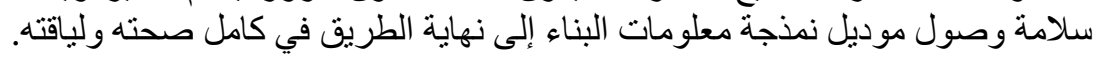

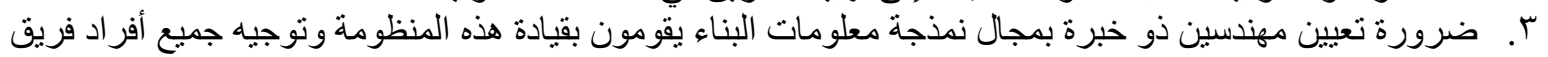

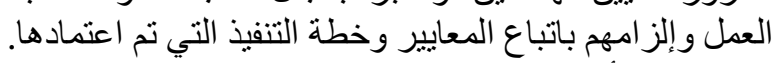

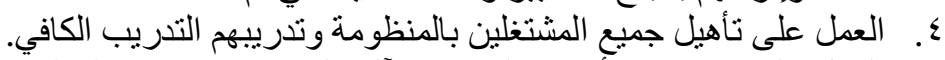

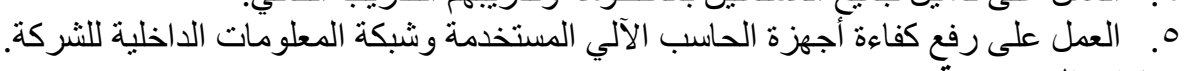
الاختصارات المستخدمة:

BIM: Building Information Modeling

BxP: BIM Execution Plan

CIC: Construction Industry Council

LOD: Level of Development

NBIMS-US: National Building Information Modeling Standards of United States 
1. BIM FORUM, (2018). BIM Project Execution Plan Guide, V 0.0, USA

2. National Institute of Building Sciences building SMART alliance, (2015), National BIM Standard - United States, V 3.0, USA

3. Computer Integrated Construction (CIC) Research Program, (2011), BIM Project Execution Planning Guide, V 2.1, USA

4. John Wiley \& Sons, Ltd., (2016). Building Information Modeling for Dummies, United Kingdom

5. Construction Industry Council (CIC), (2015). CIC Building Information Modeling Standards, Hong Kong. 